\title{
Continuous Infusion of Low-Dose 5-Fluorouracil and Radiation Therapy for Poor-Prognosis Squamous Cell Carcinoma of the Uterine Cervix
}

\author{
Charles W. Drescher, M.D., ${ }^{* 1}$ Gary C. Reid, M.D., ${ }^{*}$ Keith Terada, M.D., ${ }^{*}$ James A. Roberts, M.D., ${ }^{*}$ \\ Michael P. Hopkins, M.D. ${ }^{*}$ Claudia Perez-Tamayo, M.D. $\dagger$ and Sonja L. Schoeprel, M.D. $\dagger$ \\ ${ }^{*}$ Division of Gynecologic Oncology, Department of Obstetrics and Gynecology, and $\dagger$ Department of Radiation Oncology, The University of \\ Michigan Medical Center, Ann Arbor, Michigan 48109
}

Received July 17, 1991

\begin{abstract}
Ten patients with squamous cell carcinoma of the cervix metastatic to periaortic lymph nodes were treated with external-beam radiation therapy and synchronous infusion of intravenous 5fluorouracil (5-FU) chemotherapy at doses of $350 \mathrm{mg} / \mathrm{m}^{2} /$ day. The overall response rate was $90 \%$ with four complete responses (CR) and five partial responses (PR). The median duration of response was 11.8 months for CRs and 3.6 months for PRs. Toxicity was tolerable, with gastrointestinal symptoms and myelosuppression being noted most frequently. No patient experienced life-threatening toxicity. Median survival was 7.6 months, with only one patient being alive and free of disease at 2 years. In this pilot study we were unable to demonstrate a beneficial effect of continuous infusion of low doses of 5-FU chemotherapy concurrent with radiation therapy when compared to conventional radiotherapy in patients with advanced squamous cell carcinoma of the cervix. 1992 Academic Press, Inc.
\end{abstract}

Chemotherapy administered concurrently with radiotherapy has been reported to improve responses in a variety of tumors that respond to either therapy alone, including cancers arising in the oropharynx, esophagus, colon, and cervix [1-4]. The increased responses to therapy have been attributed to the radiosensitizing properties of certain chemotherapeutic agents. Proposed mechanisms of action for radiation sensitization include shift in tumor radiation dose-response curve, inhibition of repair of sublethal and potentially lethal radiation-induced damage, alterations in cell cycle kinetics such that an increased proportion of tumors are in a radiosensitive phase, and reduction in tumor bulk with subsequent increase in blood supply and oxygenation to irradiated tissues $[5,6]$.

5-Fluorouracil (5-FU) has received particular attention as a radiosensitizing agent in both clinical and laboratory

\footnotetext{
1 To whom reprint requests should be addressed at Swedish Hospital Medical Center, 1229 Madison St. (1050), Seattle, WA 98104.
}

studies. In vitro studies indicate that sensitization by 5FU depends on drug concentration and timing of exposure. It appears that maximum sensitization occurs when prolonged low levels of 5-FU are combined with radiotherapy $[7,8]$. The optimum schedule for clinical use is yet to be defined; however, most studies to date have utilized short, intermittent infusional therapy with relatively high doses of 5-FU [9-11]. We undertook this pilot study to evaluate the efficacy of continuous infusion of low-dose 5-FU chemotherapy administered concurrently during the course of external-beam radiation therapy in patients with squamous cell carcinoma of the cervic metastatic to periaortic lymph nodes. These patients have a 2 -year survival of approximately $20 \%$ with radiation therapy alone [12] and therefore stand to benefit significantly if a more effective treatment can be identified.

\section{MATERIALS AND METHODS}

Ten patients with squamous cell carcinoma of the cervix metastatic to periaortic nodes were treated at the University of Michigan Medical Center with combined radiotherapy and continuous infusion of 5-FU chemotherapy from January 1986 to December 1988 . Nine patients had periaortic nodal disease identified by lymphangiogram or CT scan that was histologically confirmed by needle aspiration in eight patients. Needle aspiration was unsuccessful in one patient because of obesity. Surgical debulking of enlarged nodes was not performed. Microscopically positive periaortic nodal disease was identified in the final patient with negative lymphangiogram and CT scan by retroperitoneal periaortic node sampling. All patients had large bulky pelvic tumor masses at least $6 \mathrm{~cm}$ in bidirectional diameters. Eight of ten patients had clinical stage III or IV disease and one patient had a large 
TABLE 1

FIGO Stage of Treated Patients

\begin{tabular}{cccccr}
\hline & \multicolumn{4}{c}{ FIGO stage } \\
\cline { 2 - 5 } & IA & IIB & IIIB & IVA \\
\hline No. of patients & 1 & 1 & 5 & 3 \\
\hline
\end{tabular}

pelvic recurrence $(10 \times 8 \mathrm{~cm})$ after surgical therapy for a microinvasive carcinoma (Table 1 ).

External-beam radiation therapy was delivered using 10 - or $16-\mathrm{MeV}$ linear accelerators. Daily fractions of 180 $200 \mathrm{cGy}$ were administered Monday through Friday of each week. Pelvic radiotherapy was delivered AP-PA to doses of 45-50 Gy depending upon tumor response. Pelvic portals were approximately $15 \times 15 \mathrm{~cm}$, extending to the sacroiliac joints superiorly, the obturator foramina inferiorly, and the pelvic sidewalls laterally. Periaortic nodes were treated concurrently to a dose of 44-50 Gy through midline portals encompassing nodal tissue to the bottom of T11. After completion of external-beam therapy, brachytherapy was administered using Fletcher-Suit applicators. One or two implants were employed depending on tumor geometry to boost point $\mathrm{A}$ doses to $72-88$ Gy.

$5-\mathrm{FU}, 350 \mathrm{mg} / \mathrm{m}^{2}$, dissolved in $500 \mathrm{cc}$ of D5W, was administered as a continuous intravenous infusion (CI) over $24 \mathrm{hr}$ on each day of external-beam therapy. Infusions were begun on Monday prior to the first weekly radiation treatment and completed Saturday morning 24 $\mathrm{hr}$ after the last weekly radiation treatment. Central venous access was established in all patients to facilitate administration of chemotherapy. Complete blood counts were obtained twice weekly and chemotherapy was discontinued for nutropenia less than 1000 granulocytes $/ \mathrm{mm}^{3}$ or thrombocytopenia less than $50,000 / \mathrm{mm}^{3}$. Chemotherapy was also withheld if gastrointestinal symptoms that were unresponsive to oral antiemetic or antidiarrheal therapy occurred. Radiation therapy was delayed if toxicity persisted despite discontinuation of chemotherapy. Therapy was reinstituted without dose reduction once symptoms resolved or counts recovered to

TABLE 2

Clinical Response by FIGO Stage

\begin{tabular}{lcccc}
\hline & \multicolumn{5}{c}{ Clinical response } \\
\cline { 2 - 5 } Stage & Complete & Partial & Stable & Progressive \\
\hline IA & - & 1 & - & - \\
IIB & 1 & - & - & - \\
IIIB & 1 & 4 & - & - \\
IVA & 2 & - & - & 1 \\
\hline
\end{tabular}

TABLE 3

Maximum Toxicity

\begin{tabular}{lcccc}
\hline & \multicolumn{4}{c}{ GOG grade } \\
\cline { 2 - 5 } Toxicity & 1 & 2 & 3 & 4 \\
\hline Gastrointestinal & 1 & 4 & 4 & - \\
Stromatitis & - & - & 1 & - \\
Granulocytopenia & - & 2 & 1 & - \\
Thrombocytopenia & 2 & - & - & - \\
\hline
\end{tabular}

greater that 1500 granulocytes $/ \mathrm{mm}^{3}$ or 100,000 platelets $/ \mathrm{mm}^{3}$. All toxicity was graded using standard Gynecologic Oncology Group definitions.

Patient follow-up consisted of physical examinations every 3 to 4 months. Abdominal-pelvic CAT scans were performed at the completion of therapy and approximately yearly thereafter if residual pelvic disease was not identified on physical examination. Clinical response was defined as follows: complete response (CR), disappearance of all clinically measurable disease; partial response (PR), a 50\% or greater reduction in the size and/or number of lesions; progressive disease, a $50 \%$ or greater increase in the size and/or number of lesions; stable disease, all patients not meeting any of the above criteria. All patients were followed until diseasc progression or for at least 2 years.

\section{RESULTS}

The overall response rate was $90 \%$ (Table 2). Four patients achieved a complete response and five patients demonstrated a partial response. The pelvic control rate was $40 \%$. Pelvic responses appeared to be unrelated to pelvic tumor bulk and were noted in seven of eight patients with stage III and IV disease. Mean response duration was 11.8 months for CRs and 3.6 months for PRs. Pelvic recurrences were not noted in any patient initially achieving local control.

Chemotherapy was interrupted or discontinued in five patients. Reasons for withholding chemotherapy were GI toxicity (three patients), GI toxicity and myelosuppression (one patient) and stomatitis (one patient) (Table 3). For patients not receiving the entire scheduled dose of chemotherapy, three patients received greater than $75 \%$ of the scheduled dose, one patient received $30 \%$ of the scheduled dose, and one patient received $13 \%$ of the scheduled dose. Four of nine responders (two CR, two PR) were seen in patients with chemotherapy interrupted. Radiation therapy was not interrupted in any patients. One patient required laparotomy and lysis of adhesions for small bowel obstruction 6 months after completing therapy. No patient deaths were related to treatment toxicity. 


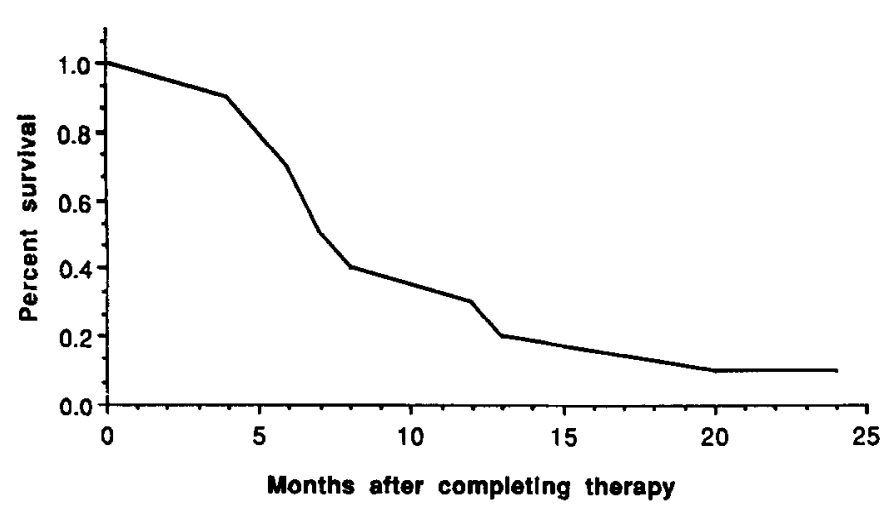

FIG. 1. Kaplan-Meier survival curve for 10 patients with cervix cancer treated with low-dose 5-FU and radiation therapy (median survival, 7.5 months).

Median survival calculated by the method of Kaplan and Meier was 7.5 months (Fig. 1). At 2 years following treatment, eight patients had died of progressive cancer and one patient remained alive and free of disease. One patient died of pneumonia 8 months after completing therapy and 2 months after laparotomy for small bowel obstruction. No tumor was identified at surgery and the patient remained clinically free of disease at the time of death. An autopsy was not performed. Metastatic tumor was identified outside the radiation treatment field in four of eight patients who died of disease including two of four patients who demonstrated CRs. Sites of distant failures included lungs, scalene and para-tracheal lymph nodes, liver, bone, and upper abdomen.

\section{DISCUSSION}

Patients with squamous cell carcinoma metastatic to the periaortic nodes respond poorly to conventional treatment and are good candidates for carefully designed experimental protocols. The treatment protocol used in this study was based on tumor cell line data reported by Byfield et al. [7], which demonstrated maximum radiosensitization when cells are continuously exposed to 5-FU at doses within the cytotoxic range. Clinical data have demonstrated that continuous infusion of 5-FU at doses near those used in this study is associated with acceptable toxicity and can achieve serum levels of 5-FU that are cytotoxic to many cell types [13-15].

The toxicity of continuous-infusion 5-FU and radiation therapy has not been thoroughly described. In this study overall toxicity was acceptable with only five episodes of grade 3 and no grade 4 toxicities noted. The development of side effects required the interruption of chemotherapy in five patients while radiation therapy was not interrupted in any patients. Three patients received at least $75 \%$ of the scheduled treatment despite interruption in therapy. To date, we have treated eight additional pa- tients with cervical adenocarcinoma or endometrial carcinoma with continuous infusion 5-FU (250-350 $\mathrm{mg} / \mathrm{m}^{2} /$ day) and radiotherapy and have observed a similar toxicity profile with three grade 3 (gastrointestinal, one; granulocytopenia, two) and no grade 4 toxicities among these patients. None of our patients have developed hand-foot syndrome, skin rashes, or cerebellar symptoms. This experience is in contrast to that of Barbounis et al. [13], Lokich et al. [14], and Huan et al. [15], who noted stomatitis and hand-foot syndrome to be among the most common complications of CI 5-FU. Patients in these series received CI 7 days/week rather than 5 days/week as used in this study. Preliminary data from the M. D. Anderson Hospital [16] have suggested that toxicity with CI 5-FU may be modulated with 5-day rather than 7-day infusions. Laparotomy for small bowel obstruction was required for one patient in the present series and future studies that include prolonged follow-up will be necessary to determine if this therapy is associated with an increased incidence of late bowel complications.

Using our treatment protocol we were unable to demonstrate improved tumor response or patient survival with CI 5-FU and radiotherapy over that with radiation therapy alone [12]. The sample size in this study is small and therefore not sensitive to minor differences between our treatment approach and conventional therapy; however, the pelvic control rate of $40 \%$ and 2-year survival of $11 \%$ noted in this study are less favorable than those in reports from many series that have utilized radiation therapy alone [17-20] making it unlikely that benefit could be demonstrated even with a larger sample size. Additionally, responses were nearly equally distributed among patients receiving the full schedule of chemotherapy and those who had chemotherapy interrupted. It might be argued that the patient with recurrent microinvasive cancer should be excluded from the response and survival data as this tumor may be biologically different from those treated at primary diagnosis; however, our results are not altered by inclusion of this patient (response rate, 90\% vs $89 \%$; median survival, 7.5 months vs 7.0 months).

Clinically the therapeutic benefit of 5-FU appears to be related to its dose or scheduling. Byfield et al. have demonstrated an increased survival in patients with advanced esophageal carcinoma when 5-FU was given in doses of $1000 \mathrm{mg} / \mathrm{m}^{2}$ daily during split-course radiation therapy [3]. Thomas et al. [9], Evans et al. [10], and Ludgate et al. [11] have demonstrated improved local control in patients with advanced gynecologic malignancies using combinations of 5-FU, mitomycin-C, and splitcourse or conventional radiation therapy, while Ludgate et al. [11] were also able to demonstrate improved patient survival. All of these studies utilized 4-day infusions of 5 -FU in the dose range of $1000 \mathrm{mg} / \mathrm{m}^{2} /$ day that were delivered once or twice during radiation therapy. Given 
our overall favorable toxicity profile, further studies might include $\mathrm{CI}$ with doses of 5-FU higher than those used in the present study or the use of other agents in addition to 5-FU. Weekly 3-day infusions of 5-FU as described by Barbounis et al. [13] may allow for dose escalation of 5$\mathrm{FU}$ greater than that in 5-day $\mathrm{CI}$ protocols. The effect of this type of schedule change on the radiosensitizing properties of 5-FU is unknown. Potential agents for combination regimens include cis-platinum, carboplatinum, and bromodeoxyuridine, which experimentally have been shown to have radiosentizing properties [21-23]. The platinum compounds are known to have significant cytotoxicity against cervical squamous cell cancer [24]. The occurrence of distant failures in our study demonstrates the need for chemotherapy combinations that utilize agents with cytotoxicity against cervical cancer. It is to be hoped that this will increase the control of micrometastatic disease, which, even in the presence of radiosensitization of known tumor deposits, may remain a major obstacle to improving patient survival.

\section{REFERENCES}

1. Fabian, C., Reddy, E., Jewell, W., Trowbridge, A. A., McCracken, D., Vogel, S., Goodwin, J., and Fletcher, W. S. Phase I-II pilot of whole abdominal radiotherapy and concomitant 5-FU as an adjuvant in colon cancer: A Southwest Oncology Group Study, Int. J. Radiat. Oncol. Biol. Phys. 15, 885-892 (1988).

2. Piver, S. M., Bacon, J. J., Vongtama, V., and Webster, J. Hydroxyurea and radiation therapy in advanced cervical cancer, $A m$. J. Obstet. Gynecol. 120, 969-972 (1974).

3. Byfield, J. E., Barone, R. M., Mendelsohn, J., Frankel, S., Quinol, L., Sharp, T., and Seagren, S. Infusional 5-fiuorouracil and $\mathrm{x}$-ray therapy for nonresectable esophageal cancer, Cancer 45, 703708 (1980).

4. Taylor, S. G., Murphy. A. K., and Stowell, T. L. Improved control in advanced head and neck cancer with simultaneous radiation and cis-platinum and 5-FU chemotherapy, Cancer Treat. Rep. 69, 933 (1985).

5. Fu, K. Biologic basis for the interaction of chemotherapeutic agents and radiation therapy, Int. J. Radiat. Oncol. Biol. Phys. 5, 21232130 (1985).

6. Russo, A., Mitchell, J., Kingell, T., Morstyn, G., and Glatstein, E. Determinants of radiosensitivity, Semin. Oncol. 12, 332-349 (1985).

7. Byfield, J. E., Calabro-Jones, P., and Klisak, I. Pharmacologic requirements for obtaining sensitization of human tumor cells in vitro to combined 5-fluorouracil or ftorafur and x-rays, Int. J. Radiat. Oncol. Biol. Phys. 8, 1923-1933 (1982).

8. Looney, W. B., Hopkins, H. A., MacLeod, M. S., and Ritenour, R. Solid tumor models for the assessment of different treatment modalities. XII. Combined chemotherapy-radiotherapy: Variation of time interval between time of administration of 5-fluorouracil and radiation and its effect on the control of tumor growth, Cancer 44, 437-445 (1979).

9. Thomas, G., Dembo, A., Beale, F., Bean, H., Bush, R., Herman, J., Pringle, J., Rawlings, G., Sturgeon, J., Fine, S., and
Black, B. Concurrent radiation, mitomycin-C and 5-fluorouracil in poor prognosis carcinoma of cervix: Preliminary results of a phase I-II study, Int. J. Radiat. Oncol. Biol. Phys. 10, 1785-1790 (1984).

10. Evans, I. S., Kersh, C. R., Constable, W. C., and Taylor, P. T. Concomitant 5-fluorouracil, mitomycin-C, and radioatherapy for advanced gynecologic malignancies, Int. J. Radiat. Oncol. Biol. Phys. 15, 901-906 (1988).

11. Ludgate, S. M., Crandon, A. J., Walker, Q., and Langlands, A. O. Synchronous 5-fluorouracil, mitomycin-C and radiation therapy in the treatment of locally advanced carcinoma of the cervix, Int. J. Radiat. Oncol. Biol. Phys. 15, 893-899 (1988).

12. Shingleton, H. M., and Orr, J. W. Eds. In Cancer of the cervix: Diagnosis and treatment, Churchill/Livingston, New York, p. 191 (1987).

13. Barbounis, V. P., Kalofonos, H. P., Munro, A. J., McKenzie, C. G., Sackier, J. M., and Epentos, A. A. Treatment of colorectal cancer and other malignancies with continuous infusion of 5-fluorouracil, Anticancer Res. 9, 33-44 (1989).

14. Lokich, J., Bothe, A., Fine, N., and Perri, J. Phase I study of protracted venous infusion of 5-fluorouracil, Cancer 48, 2565-2568 (1981).

15. Huan, S., Pazdur, R., Singhakowinta, A., Samal, B., and Vaitkevicius, V. K. Low-dose continuous infusion 5-fluorouracil, evaluation in advanced breast cancer, Cancer 63, 419-422 (1989).

16. Rich, T. A., Hughes, L., Ajani, J. A., and Brock, W. Low dose, continuous infusion 5-fluorouracil plus radiotherapy for anal cancer, Int. J. Radiat. Oncol. Biol. Phys. 18, 710 (1990).

17. Nelson, J. H., Boyce, J., Macasaet, M., Lu, T., Bohorquez, J. F., Nicastri, A. D., and Fruchter, R. Incidence, significance and followup of para-aortic lymph node metastases in late invasive carcinoma of the cervix, Am. J. Obstet. Gynecol. 128, 336-340 (1977).

18. Brookland, R. K., Rubin, S., and Danoff, B. F. Extended field irradiation in the treatment of patients with cervical carcinoma involving biopsy proven para-aortic nodes, Int. J. Radiat. Oncol. Biol. Phys. 10, 1875-1879 (1984).

19. Potish, R. A., Twiggs, L. B., Prem, K. A., Levitt, S. H., and Adcock, L. L. The impact of extraperitoneal surgical staging on morbidity and tumor recurrence following radiotherapy for cervical cancer, Am. J. Clin. Oncol. 7, 241-251 (1984).

20. Komaki, R., Mattingly, R. F., Hoffman, R. G., Barber, S. W., Satre, R., and Greenber, M. Irradiation of para-aortic lymph node metastases from carcinoma of the cervix or endometrium. Preliminary results, Radiology 147, 245-248 (1983).

21. DeWit, L. Combined treatment of radiation and cis-diamminedichloroplatinum (II): A review of experimental and clinical data, Int. J. Radiat. Oncol. Biol. Phys. 13, 403-417 (1987).

22. Eisenberger, M., Jacobs, M., Sinibaldi, V., Gray, W., and Elias, E. G. Simultaneous treatment with carboplatin and conventional radiation for patients with unresectable squamous cell carcinoma of the head and neck: Preliminary phase I and II results, in Carboplatin current perspectives and future directions (P. A. Bunne, Ed.), Saunders, Philadelphia (1990).

23. Kinsella, T. J., Russo, A., Mitchell, J. B., Rowland, J., Jenkins, J., Schwade, J., Myers, C. E., Collins, J. M., Speyer, J., Kornblith, P., Smith, B., Kufta, C., and Glatstein, E. A phase I study of intermittent intravenous bromodeoxyuridine (BUdR) with conventional fractionated irradiation, Int. J. Radiat. Oncol. Biol. Phys. 10, 69-76 (1984).

24. Thigpen, T., Vance, R. B., Balducci, L., and Blessing, J. Chemotherapy in the management of advanced or recurrent cervical and endometrial carcinoma, Cancer 48, 658-665 (1981). 